\section{Submission of typescripts}

Two copies of the manuscript should be submitted to one of the Executive Editors (addresses on outside front cover). Papers may also be submitted electronically to those Executive Editors with electronic mail addresses. The editor will acknowledge receipt of the manuscripts. It is important that authors inform the editor of any changes of address whilst their paper is under consideration.

Submission of a paper is taken to imply that it has not been previously published and that it is not being considered for publication elsewhere. Upon acceptance of a paper, the author will be asked to transfer copyright to the publisher.

\section{Typescript}

Papers should be typed, double-spaced, on one side only and with generous margins. The pages must be numbered.

The first page should give the title, the author's name and institution, and a short abstract intelligible to mathematicians.

The title, while brief, must be informative (e.g. $A$ new proof of the ergodic theorem, whereas Some applications of a theorem of Birkhoff would be useless).

\section{Notation}

It is important that mathematicians expressions are clear to a printer (who is not a mathematician). For instance, $n_{k}(n$ sub $k$ ) is common usage, but avoid if possible using $c$ sub $n$ sub $k$. Fractions are generally best expressed by a solidus. Complicated exponents like

$$
\exp \left\{z^{2} \sin \theta /\left(1+y^{2}\right)\right\}
$$

should be shown in this and no other way.

In the typescript, italics, small capitals and capitals are specified by single, double and triple underlining. Bold-faced types is shown by wavy underlining.

It helps if displayed equations or statements which will be quoted later are numbered in order on the right of their line. They can then be referred to by, for example, 'from (7)'.

The author must enable the printer (if necessary by pencilled notes in the margin) to distinguish between similar symbols such as $o, O, \mathrm{o}, \mathrm{O}, 0 ; x, \mathrm{X}, \times ; \phi, \Phi$, $\varnothing ; 1,1 ; \varepsilon, \epsilon ; \kappa, k$.

There is no need to underline Greek or script letters provided these are clearly typed. Any special symbols should be explained on a separate sheet of directions for the printer.

If an author wishes to mark the end of the proof of a theorem, the sign $\square$ may be used.

Footnotes should be avoided.

\section{Diagrams}

Figures and drawings should be on separate sheets in black ink or produced by computer to comparable quality. Photocopies are acceptable only if they are as clear as the originals. Symbols, legends and captions should be given on a transparent overlay. Each text figure must be numbered as Figure 1, Figure $2, \ldots$ and its intended position clearly indicated in the typescript. The author's name in pencil must be on all separate sheets of diagrams.

Figures should be used sparingly and only when they greatly clarify the exposition.

The publisher recognizes that some authors do not have the facilities for producing drawings of a sufficiently high standard to be reproduced directly and is therefore willing to have such diagrams re-drawn, provided that they are clear.

\section{Tables}

Tables should be numbered (above the table) and set out on separate sheets. Indicate the position of each in the text as for figures.

\section{References}

References should be collected at the end of the paper numbered in alphabetical order of the author's names. A reference to a book should give the title, in italics, and then in roman type the publisher's name and the place and year of publication;

[4] N. Dunford \& J. T. Schwartz Linear Operators Part I. Wiley: New York, 1958.

A reference to a paper should give in italics the title of the periodical, the number of the volume and year, and the beginning and end pages of the paper. Titles should be abbreviated as in Mathematical Reviews:

[6] J. E. Littlewood. The 'pits effect' for functions in the unit circle. J. Analyse Math. 23 (1970), 236-268.

\section{Proofs}

Authors receive one set of proofs for correction. If excessive alterations to the original manuscript are requested after the paper has been typeset, the author will be charged the cost of resetting. For papers with more than one author the proofs are sent to the first named author unless the editor receives other instructions. It is important that proofs are corrected and returned promptly.

\section{Offprints}

50 offprints of each article will be supplied free to each first named author. Extra offprints may be purchased from the publisher. 


\section{Ergodic theory and dynamical systems}

\section{VOLUME 16 PART 1 FEBRUARY 1996}

\section{CONTENTS}

Adl-Zarabi, K. Absolutely continuous invariant measures for piecewise expanding $C^{2}$ transformations in $R^{n}$ on domains with cusps on the boundaries

Chernov, N. I. and Haskell, C. Nonuniformly hyperbolic K-systems are Bernoulli

Fagnani, F. Some results on the classification of expansive automorphisms of compact abelian groups

Forni, G. Construction of invariant measures supported within the gaps of Aubry-Mather sets

Françoise, J. P. Successive derivatives of a first return map, application to the study of quadratic vector fields

Goodson, G. R. et al. Ergodic transformations conjugate to their inverses by involutions

Makarov, N. and Smirnov, S. Phase transition in subhyperbolic Julia sets 125

Vargas, E. Measure of minimal sets of polymodal maps

Walters, $P$. Topological Wiener-Wintner ergodic theorems and a random $L^{2}$ ergodic theorem

\section{CAMBRIDGE}

